\title{
Erratum to: Dipeptidyl Peptidase 4-Deficient Rats Have Improved Bile Secretory Function in High Fat Diet-Induced Steatosis
}

\author{
Shani Ben-Shlomo • Isabel Zvibel • Liane Rabinowich • \\ Ilana Goldiner · Amir Shlomai • Erwin M. Santo • \\ Zamir Halpern · Ran Oren · Sigal Fishman
}

Published online: 23 November 2012

(C) Springer Science+Business Media New York 2012

\section{Erratum to: Dig Dis Sci}

DOI 10.1007/s10620-012-2353-7

There is an error noticed in the first author's last name, It is published as Shlomo instead of Ben-Shlomo.

The online version of the original article can be found under doi:10.1007/s10620-012-2353-7.

S. Ben-Shlomo · I. Zvibel · L. Rabinowich - A. Shlomai ·

E. M. Santo $\cdot$ Z. Halpern $\cdot$ S. Fishman

The Research Center for Digestive Tract and Liver Diseases,

Tel-Aviv Sourasky Medical Center, Tel-Aviv, Israel

e-mail: shani.benshlomo@gmail.com

I. Zvibel

e-mail: isab@tasmc.health.gov.il

L. Rabinowich

e-mail: liane.rabinowich@gmail.com

A. Shlomai

e-mail: amirsh@tasmc.health.gov.il

E. M. Santo

e-mail: erwins@tasmc.health.gov.il

Z. Halpern

e-mail: halpernza@tasmc.health.gov.il

S. Ben-Shlomo - I. Zvibel · L. Rabinowich · A. Shlomai ·

E. M. Santo $\cdot$ Z. Halpern $\cdot$ S. Fishman

The Sackler Faculty of Medicine, Tel-Aviv University,

Tel-Aviv, Israel
I. Goldiner

Central Biochemistry Lab, Tel-Aviv Sourasky Medical Center, Tel-Aviv, Israel

e-mail: ilanag@tasmc.health.gov.il

R. Oren

Gastroenterology Institute, Hadassah Medical Center, Jerusalem, Israel

e-mail: ranoren@hadassah.org.il

S. Fishman $(\square)$

Gastroenterology Department, Tel Aviv Sourasky Medical

Center, 6 Weizmann, Tel Aviv 64239, Israel

e-mail: sigalf@tasmc.health.gov.il 